\title{
The Long Time Behavior of Solutions for a Generalized Boussinesq System
}

\author{
Shaoyong Lai Hongmei Cai \\ Department of Applied Math., Southwestern Univ. of Finance and Economics, Chengdu 610074, China
}

\begin{abstract}
The initial-boundary value problem is investigated for a generalized Boussinesq equation with the quadratic nonlinearity. For small initial data and homogeneous boundary conditions, its solution is constructed in the form of a series which converges absolutely and uniformly. The long time asymptotic expansion of the solution is acquired to show the nonlinear effects of amplitude.
\end{abstract}

Keywords: Boussinesq equation, Well-posedness, Fourier transform, Exponential decay

\section{Introduction}

Boussinesq equation was first presented in [1] to research the propagation of small amplitude's long wave on the surface of shallow water. From then on, people have made a great deal of investigations for the equation (see[2]-[4]). One of the classical Boussinesq equations is written in the form

$$
u_{t t}=-\alpha u_{x x x x}+u_{x x}+\beta\left(u^{2}\right)_{x x},
$$

where constant coefficients $\alpha$ and $\beta$ depend on the depth of fluid and the speed of waves. Clarkson [3] established a general method to determine the exact solutions of equation (1). Hirota deduced conservation laws and examined it's numerical solutions in literature [5]. Lai [9]-[10] studied the long time behavior of solutions for nonlinear wave equations. Nakamura [6] studied the exponential decay of the Boussinesq equation with spherical symmetry.

Varlamov [7] considered the following Boussinesq equation with initial-boundary assumptions

$$
u_{t t}-2 b u_{t x x}=-\alpha u_{x x x x}+u_{x x}+\beta\left(u^{2}\right)_{x x},
$$

where $\beta \in R^{1}, \alpha$ and $b$ are positive constants and $\alpha>b^{2}$. Making use of the eigenfunction expansion method in a ball, Varlamov [8] investigated a long time asympotics of solution for a Boussinesq equation similar to equation (2). Polat [11] considered the blow up phenomena of solutions for the following Boussinesq equation with damping term

$u_{t t}-u_{x x}+\delta u_{x x x x}-\lambda u_{x x t t}-r u_{x x t}=\beta\left(u^{2}\right)_{x x}-\gamma^{2} u$,

where $\delta, \lambda, r, \beta$ and $\gamma$ are constants, which satisfy some assumptions.

The objective of this paper is to study the wellposedness of the following generalized Boussinesq equation with initial-boundary conditions

$$
\begin{array}{r}
u_{t t}-a u_{t t x x}-2 b u_{t x x}+d u=-c u_{x x x x}+u_{x x}+ \\
\beta\left(u^{2}\right)_{x x}+h u^{2},
\end{array}
$$

where $a>0, b>0, c>0 d \geqslant 0, h$ and $\beta$ are constants and $a+c>b^{2}$. Under some assumptions, the existence and the uniqueness of solution for equation (3) are established. It will be shown that the long time behavior of the solution in equation shows the presence of damped oscillations decaying exponentially in time as $t \rightarrow \infty$. The methods for the proof of our main theorem in this paper are based on those of [7]. However, it should be emphasized that the technique for proving uniqueness of the solution is different from that presented in [7] in which the time extension method was used.

\section{Main result}

The task of this paper is to consider the following generalized Boussinesq system with initialboundary conditions

$$
\left\{\begin{array}{l}
u_{t t}-a u_{t t x x}-2 b u_{t x x}+d u=-c u_{x x x x}+ \\
u_{x x}+\beta\left(u^{2}\right)_{x x}+h u^{2} \\
u(0, t)=u(\pi, t)=0 \\
u_{x x}(0, t)=u_{x x}(\pi, t)=0 \\
u(x, 0)=\varepsilon^{2} \varphi(x), u_{t}(x, 0)=\epsilon^{2} \psi(x)
\end{array}\right.
$$

where $a>0, d \geqslant 0, b, c$ and $\varepsilon$ are positive constants, $x \in(0, \pi), t>0, h$ and $\beta \in R^{1}$.

Definition 2.1: If $u(0)=u(\pi)=u^{\prime \prime}(0)=$ $u^{\prime \prime}(\pi)=\ldots=u^{2 n-2}(0)=u^{2 n-2}(\pi)=0$ and 
$u^{(2 n)}(x) \in L^{2}(0, \pi)$, the function $u(x)$ is said to belong to the class $C^{2 n}(0, \pi), n \geqslant 1$.

Definition 2.2: The function $u(x, t)$ defined on $[0, \pi] \times[0,+\infty)$ is said to be the classical solution of the problem defined by system $(4)$, if $u(x, t)$ and its derivatives included in (4) are bounded and continuous, and satisfy system (4).

Theorem 2.3: If $a>0, c>0, d \geqslant 0, a+c>$ $b^{2}, \varphi(x) \in C^{6}(0, \pi), \psi(x) \in C^{4}(0, \pi)$, there is a $\varepsilon_{0}$, for any $\varepsilon$ satisfying $0<\varepsilon<\varepsilon_{0}$, problem (4) has a unique classical solution expressed in the form

$$
u(x, t)=\sum_{N=1}^{\infty} \varepsilon^{N+1} u^{(N)}(x, t),
$$

where $u^{(N)}(x, t)$ will be defined in the proof ( $\operatorname{see}(23)$ or (24)). Series (5) and its derivatives, which appear in problem (4), converge absolutely and uniformly. For $x \in[0, \pi], t \geqslant 0,0<\varepsilon<\varepsilon_{0}$. The problem (5) has the following asymtotics as $t$ is sufficiently large

$$
\begin{array}{r}
u(x, t)=e^{-\frac{b}{1+a} t}[(A \cos \sigma t+B \sin \sigma t) \sin x+ \\
\left.O\left(e^{\left[\frac{\eta b}{1+a} t\right]}\right)\right],
\end{array}
$$

where $\sigma=\frac{\sqrt{a c+a+c-b^{2}+1+a d+d}}{1+a}$ is a positive constant, $0<\eta<\frac{3}{1+4 a}$.

For simplicity, throughout the paper, we denote by $\mathrm{C}$ any possitive constants independent of $t$, which may depend on $\varphi(x), \psi(x)$ and other constants appearing in system (4).

\section{Proof of Theorem 2.3}

\subsection{Existence of solution}

We make an odd extension for $x$ on $[-\pi, 0]$, and represent $u(x, t)$ in the form of Fourier series expressed by

$$
u(x, t)=\sum_{n=-\infty, n \neq 0}^{\infty} \widehat{u}_{n}(t) e^{i n x}
$$

where

$$
\widehat{u}_{n}(t)=\frac{1}{2 \pi} \int_{-\pi}^{\pi} u(x, t) e^{i n x} d x
$$

from which we have $\widehat{u}_{-n}(t)=-\widehat{u}_{n}(t), n \geqslant 1$.

In the sequel we shall denote the norm of the space of functions belonging to $L^{2}(-\pi, \pi)$ for each fixed $t>0$,

$$
\|u(t)\|=\|u(t)\|_{L^{2}(-\pi, \pi)}=\left(\int_{-\pi}^{\pi}|u(x, t)|^{2} d x\right)^{\frac{1}{2}} .
$$

It follows from (7) that

$$
\begin{array}{r}
u(x, t)=2 i \sum_{n=1}^{\infty} \widehat{u}_{n}(t) \sin n x, \\
\widehat{u}_{n}(t)=\frac{1}{i \pi} \int_{0}^{\pi} u(x, t) \sin n x d x .
\end{array}
$$

Noting the initial functions on $[-\pi, \pi]$, which have $\widehat{\varphi}_{-n}=-\widehat{\varphi}_{n}, \widehat{\psi}_{-n}=-\widehat{\psi}_{n}, n \geqslant 1$, we get

$$
\begin{aligned}
& \varphi(x)=\sum_{n=-\infty, n \neq 0}^{\infty} \widehat{\varphi}_{n} e^{i n x}, \\
& \psi(x)=\sum_{n=-\infty, n \neq 0}^{\infty} \widehat{\psi}_{n} e^{i n x} .
\end{aligned}
$$

Furthermore on $[0, \pi]$, we obtain

$$
\left\{\begin{array}{l}
\varphi(x)=2 i \sum_{n=1}^{\infty} \widehat{\varphi}_{n} \sin n x \\
\widehat{\varphi}_{n}=\frac{1}{i \pi} \int_{0}^{n=1} \varphi(x) \sin n x d x \\
\psi(x)=2 i \sum_{n=1}^{\infty} \widehat{\psi}_{n} \sin n x \\
\widehat{\psi}_{n}=\frac{1}{i \pi} \int_{0}^{\pi} \psi(x) \sin n x d x
\end{array}\right.
$$

Integrating (11) and using the smoothness assumption of initial data yield the following inequalities

$$
\left|\widehat{\varphi}_{n}\right| \leqslant C_{1} n^{-6}, \quad\left|\widehat{\psi}_{n}\right| \leqslant C_{1} n^{-4}, \quad n \geqslant 1,
$$

where $C_{1}$ is a positive constant.

Substituting (7), (9) and (10) into (4) gives rise to the following Cauchy problem for $\widehat{u}_{n}(t)$

$$
\left\{\begin{array}{l}
\left(1+a n^{2}\right) \widehat{u}_{n}^{\prime \prime}(t)+2 b n^{2} \widehat{u}_{n}^{\prime}(t)+ \\
\left(c n^{4}+n^{2}+d\right) \widehat{u}_{n}(t) \\
=\left(-\beta n^{2}+h\right) p\left(\widehat{u}_{n}(t)\right) \\
\widehat{u}_{n}(0)=\varepsilon^{2} \widehat{\varphi}_{n}, \quad \widehat{u}_{n}^{\prime}(t)=\varepsilon^{2} \widehat{\psi}_{n}
\end{array}\right.
$$

where

$$
\begin{aligned}
p\left(\widehat{u}_{n}(t)\right) & =\sum_{g=-\infty, g \neq 0, n}^{\infty} \widehat{u}_{n-g}(t) \widehat{u}_{g}(t), \\
\widehat{u}_{-n}(t) & =-\widehat{u}_{n}(t), \quad n \geqslant 1 .
\end{aligned}
$$

If $n=1$, it has

$$
\begin{aligned}
p\left(\widehat{u}_{1}(t)\right) & =2 \sum_{g=1}^{\infty} \widehat{u}_{-g}(t) \widehat{u}_{1+g}(t) \\
& =-2 \sum_{g=1}^{\infty} \widehat{u}_{g}(t) \widehat{u}_{1+g}(t) .
\end{aligned}
$$

If $n \geqslant 2$, we have

$$
p\left(\widehat{u}_{n}(t)\right)=\sum_{g=1}^{n-1} \widehat{u}_{n-g}(t) \widehat{u}_{g}(t)-2 \sum_{g=1}^{\infty} \widehat{u}_{g}(t) \widehat{u}_{n+g}(t) .
$$


Setting $\Phi_{n}=\varepsilon \widehat{\varphi}_{n}$ and $\Psi_{n}=\varepsilon \widehat{\psi}_{n}$, we get the solution formula for problem (13) in the form

$$
\begin{aligned}
\widehat{u}_{n}(t) & =\varepsilon e^{\frac{-b n^{2} t}{1+a n^{2}}}\left\{\left[\cos \left(\sigma_{n} t\right)\right.\right. \\
& \left.\left.+\frac{b n^{2}}{1+a n^{2}} \frac{\sin \left(\sigma_{n} t\right)}{\sigma_{n}}\right] \Phi_{n}+\frac{\sin \left(\sigma_{n} t\right)}{\sigma_{n}} \Psi_{n}\right\} \\
& -\frac{\beta n^{2}-h}{\sigma_{n}\left(1+a n^{2}\right)} K,
\end{aligned}
$$

where

$$
\begin{gathered}
K=\int_{0}^{t} \exp \left[\frac{-b n^{2}}{1+a n^{2}}(t-\tau)\right] \sin \left[\sigma_{n}(t-\tau)\right] p\left(\widehat{u}_{n}(t)\right) d \tau \\
\sigma_{n}=\frac{\sqrt{a c n^{6}+\left(a+c-b^{2}\right) n^{4}+(1+a d) n^{2}+d}}{1+a n^{2}}
\end{gathered}
$$

in which we require $a+c>b^{2}, d \geqslant 0$.

Now, we consider integral equation (16) by using perturbation technique. Firstly, we express $\widehat{u}_{n}(t),(n \geqslant 1)$ as a form of series about $\varepsilon$

$$
\widehat{u}_{n}(t)=\sum_{N=1}^{\infty} \varepsilon^{N+1} \widehat{\xi}_{n}^{(N)}(t) .
$$

Substituting (17) into (16) and equating the coefficients of like powers of $\varepsilon$ result in the following formula.

When $N=0$, we have

$$
\begin{array}{r}
\widehat{\xi}_{n}^{(0)}(t)=\varepsilon e^{\frac{-b n^{2} t}{1+a n^{2}}}\left\{\left[\cos \left(\sigma_{n} t\right)+\right.\right. \\
\left.\left.\frac{b n^{2}}{1+a n^{2}} \frac{\sin \left(\sigma_{n} t\right)}{\sigma_{n}}\right] \Phi_{n}+\frac{\sin \left(\sigma_{n} t\right)}{\sigma_{n}} \Psi_{n}\right\} .
\end{array}
$$

When $N \geqslant 1$, we get

$$
\begin{aligned}
& \widehat{\xi}_{n}^{(N)}(t)=\frac{\beta n^{2}+h}{\sigma_{n}\left(1+a n^{2}\right)} \int_{0}^{t} \exp \left[\frac{-b n^{2}}{1+a n^{2}}(t-\tau)\right] \\
& \sin \left[\sigma_{n}(t-\tau)\right] Q_{N}\left(\widehat{\xi}_{n}^{(j)}(\tau)\right) d \tau
\end{aligned}
$$

where $n \geqslant 1$ and

$$
\begin{aligned}
Q_{N}\left(\widehat{\xi}_{n}^{(j)}(\tau)\right)= & \varepsilon_{n} \sum_{g=1}^{n-1} \sum_{j=1}^{N} \widehat{\xi}_{n-g}^{(j-1)}(\tau) \widehat{\xi}_{g}^{(N-j)}(\tau) \\
& -2 \sum_{g=1}^{\infty} \sum_{j=1}^{N} \widehat{\xi}_{n+g}^{(j-1)}(\tau) \widehat{\xi}_{g}^{(N-j)}(\tau)
\end{aligned}
$$

$\varepsilon_{1}=0 ; \varepsilon_{n}=1$, if $n \geqslant 2$.

In order to state that formula (7) represents the solution of problem (4), we need to prove that the series

$$
\begin{aligned}
u(x, t) & =\sum_{n=-\infty, n \neq 0}^{\infty} e^{i n x} \sum_{N=0}^{\infty} \varepsilon^{N+1}{\widehat{\xi_{n}}}^{(N)}(t) \\
& =2 i \sum_{n=1}^{\infty} \sin n x \sum_{N=0}^{\infty} \varepsilon^{N+1} \widehat{\xi}_{n}^{(N)}(t)
\end{aligned}
$$

converges absolutely and uniformly. To do this, we construct the following estimate for $n \geqslant 1, t>$ $0, N>0$,

$$
\left|\widehat{\xi}_{n}^{(N)}(t)\right| \leqslant C^{N}(N+1)^{-2} n^{-6} e^{-\frac{b}{1+a} t},
$$

where $C$ is a positive constant independent of $N, n, \varepsilon$ and $t$. We will use the induction method to prove inequality $(20)$.

When $N=0$, form (18), we have

$$
\begin{aligned}
\left|\widehat{\xi}_{n}^{(0)}(t)\right| & \leqslant \varepsilon e^{\frac{-b n^{2} t}{1+a n^{2}}}\left[\left(1+\frac{b n^{2}}{\sigma_{n}\left(1+a n^{2}\right)}\right)\left|\Phi_{n}\right|\right. \\
& \left.+\frac{1}{\sigma_{n}}\left|\Psi_{n}\right|\right] \\
& \leqslant \varepsilon n^{-6} e^{-\frac{b}{1+a} t} .
\end{aligned}
$$

Assuming that $(20)$ is valid for all ${\widehat{\xi_{n}}}^{(s)}(t)$ with $0 \leqslant$ $s \leqslant N-1$, we shall prove that (20) also holds for $s=N$. According to [7], for any integer $n \geqslant 1, g \geqslant$ 1 , and $g \neq n$, we have

$$
\begin{gathered}
|n-g|^{-6} g^{-6} \leqslant 2^{6} n^{-6}\left[g^{-6}+|n-g|^{-6}\right] \\
j^{-2}(N+1-j)^{-2} \leqslant 2^{2}(N+1)^{-2}\left[j^{-2}+(N+1-j)^{-2}\right] .
\end{gathered}
$$

Using $\frac{b n^{2}}{1+a n^{2}} \geqslant \frac{b}{1+a}(n \neq 0)$ and (19) leads to

$$
\begin{aligned}
& \left|\widehat{\xi}_{n}^{(N)}(t)\right| \leqslant C|\beta|(N+1)^{-2} n^{-6} \\
& \times \sum_{g=1}^{\infty}\left(g^{-6}+|n-g|^{-6}\right) \\
& \times \sum_{j=1}^{N} C^{j-1} C^{N-j}\left[j^{-2}+(N+1-j)^{-2}\right] \\
& \times\left|S_{N}(n, t)\right|
\end{aligned}
$$

where

$$
\begin{aligned}
& \left|S_{N}(n, t)\right| \\
\leqslant & C e^{\frac{-b n^{2}}{1+a n^{2}} t} \int_{0}^{t} \exp \left(\frac{b n^{2}}{1+a n^{2}}-\frac{2 b}{1+a} \tau\right) d \tau \\
= & C e^{-\frac{b n^{2}}{1+a n^{2}} t} \times\left|\frac{\left.e^{\left(\frac{b n^{2}}{1+a n^{2}}-\frac{2 b}{1+a}\right.}\right) \tau-1}{\frac{b n^{2}}{1+a n^{2}}-\frac{2 b}{1+a}}\right| \\
\leqslant & C e^{-\frac{b}{1+a} t} .
\end{aligned}
$$


Therefore (20) holds.

For $n \geqslant 2$, we derive

$$
\frac{b n^{2}}{1+a n^{2}} \geqslant \frac{(1+\eta) b}{1+a}
$$

where $0<\eta<\frac{3}{1+4 a}$. Furthermore, we get

$$
\left|\widehat{\xi}_{n}^{(N)}(t)\right| \leqslant C^{N}(N+1)^{-2} n^{-6} e^{-\frac{1+\eta b}{1+a} t} .
$$

Substituting (17) into (8) and interchanging the order of summation in the series, for $x \in[0, \pi], t \geqslant$ $0, \varepsilon \in\left[0, \varepsilon_{0}\right]$, we get

$$
\begin{aligned}
u(x, t) & =2 i \sum_{n=1}^{\infty} \widehat{u}_{n}(t) \sin n x \\
& =2 i \sum_{n=1}^{\infty} \sin n x \sum_{N=0}^{\infty} \varepsilon^{N+1}{\widehat{\xi_{n}}}^{(N)}(t) \\
& =\sum_{N=0}^{\infty} \varepsilon^{N+1} u^{(N)}(x, t)
\end{aligned}
$$

where

$$
u^{(N)}(x, t)=2 i \sum_{n=1}^{\infty} \widehat{\xi}_{n}^{(N)}(t) \sin n x .
$$

Differentiating (18)-(19), for $k=1,2$, we have

$$
\begin{aligned}
& \partial_{t}^{k} \widehat{\xi}_{n}^{(0)}(t)=\sum_{l=0}^{k} c_{k}^{l}(-1)^{l}\left(\frac{b n^{2}}{1+a n^{2}}\right)^{l} e^{\frac{-b n^{2} t}{1+a n^{2}}} \times \\
& \partial_{t}^{k-l}\left\{\left[\cos \left(\sigma_{n} t\right)+\frac{b n^{2}}{1+a n^{2}} \frac{\sin \left(\sigma_{n} t\right)}{\sigma_{n}}\right] \Phi_{n}\right. \\
& \left.+\frac{\sin \left(\sigma_{n} t\right)}{\sigma_{n}} \Psi_{n}\right\}, \\
& \partial_{t}^{k} \widehat{\xi}_{n}^{(N)}(t)=-\frac{\beta n^{2}-h}{\sigma_{n}\left(1+a n^{2}\right)} \times \\
& \int_{0}^{t} g_{k}(n, t-\tau) Q_{N}\left(\widehat{\xi}_{n}^{(j)}(\tau)\right) d \tau+R_{k}(n, t),
\end{aligned}
$$

where

$$
\begin{aligned}
& g_{k}(n, t)=\sum_{l=0}^{k} c_{k}^{l}(-1)^{l}\left(\frac{b n^{2}}{1+a n^{2}}\right)^{l} e^{\frac{-b n^{2}}{1+a n^{2}} t} \sigma_{n}^{k-l} \\
& \times \sin \left[\sigma_{n} t+\frac{(k-l) \pi}{2}\right],
\end{aligned}
$$

$Q_{N}\left({\widehat{\xi_{n}}}^{(j)}(\tau)\right)$ is defined by (19) and $c_{k}^{l}$ are binomial coefficients. $R_{k}(n, t)$ are defined as follows

$R_{1}(n, t)=0, \quad R_{2}(n, t)=-\frac{\beta n^{2}-h}{1+a n^{2}} Q_{N}\left(\widehat{\xi}_{n}^{(j)}(\tau)\right)$.
By the bounded properties of $\frac{b n^{2}}{1+a n^{2}}$ and $\frac{\beta n^{2}}{\sigma_{n}\left(1+a n^{2}\right)}$ and making the estimates similar to (20) for $n \geqslant 1, N \geqslant 0, t>0, k \geqslant 0,1,2$, we know that the following inequalities hold

$$
\begin{gathered}
\left|\partial_{t}^{k} \widehat{\xi}_{n}^{(N)}(t)\right| \leqslant C^{N}(N+1)^{-2} n^{k-6} e^{-\frac{b}{1+a} t}, \\
\left|\partial_{t}^{k} \widehat{u}_{n}^{(N)}(t)\right| \leqslant C n^{k-6} e^{-\frac{b}{1+a} t} .
\end{gathered}
$$

The $u_{x x x x}$ and $u_{t t x x}$ are the highest order of derivative terms appearing in system (4). Inequalities (25) and (26) show that the derivatives of $u(x, t)$ in problem (4) are absolutely and uniformly convergent. Thus, we know $u(x, t)$ is a classical solution of problem (4).

\subsection{Uniqueness of the solution}

Assuming that problem (4) has two classical solutions $u^{(1)}(x, t)$ and $u^{(2)}(x, t)$, we shall prove that $u^{(1)}(x, t)$ is equal to $u^{(2)}(x, t)$. Making an odd extension for the two solutions on $(-\pi, 0]$, we notice that the two solutions belong to space $L^{2}(-\pi, \pi)$. According to Definition 2.2, for each fixed $t>0$, we have

$\max _{x \in[-\pi, \pi]}\left|u^{(1)}(x, t)\right|<c_{t}, \max _{x \in[-\pi, \pi]}\left|u^{(2)}(x, t)\right|<c_{t}$,

where $c_{t}$ is a constant depending on $t$.

Setting $w(x, t)=u^{(1)}(x, t)-u^{(2)}(x, t)$ and making an even extension for $w(x, t)$ on $\ldots(-3 \pi,-2 \pi)$, $(-2 \pi,-\pi),(\pi, 2 \pi),(2 \pi, 3 \pi), \ldots$, from $(4)$, we have

$$
\begin{aligned}
& w_{t t}-a w_{t t x x}-2 b w_{t x x}+d w=-c w_{x x x x}+w_{x x} \\
& +\beta\left[w(x, t)\left(u^{(1)}(x, t)+u^{(2)}(x, t)\right)\right]_{x x} \\
& +h w(x, t)\left[u^{(1)}(x, t)+u^{(2)}(x, t)\right] \\
& w(x, 0)=w_{t}(x, 0)=0 .
\end{aligned}
$$

Taking the Fourier transform of $w$ on $(-\infty,+\infty)$, namely,

$$
\widehat{w}(\xi, t)=\int_{-\infty}^{+\infty} w(x, t) e^{-i \xi x} d x
$$

we obtain

$$
\begin{array}{r}
\left(1+a \xi^{2}\right) \widehat{w}^{\prime \prime}(\xi, t)+2 b \xi^{2} \widehat{w}^{\prime}(\xi, t)+ \\
\left(c \xi^{4}+\xi^{2}+d\right) \widehat{w}(\xi, t)=\left(-\beta \xi^{2}+h\right) \widehat{f}(\xi, t),
\end{array}
$$

where $\widehat{f}(\xi, t)=w\left(u^{(1)}+u^{(2)}\right)(x, t)$. It follows from (27) that

$$
\begin{gathered}
\widehat{w}(\xi, t)=-\frac{\beta \xi^{2}-h}{\sigma_{\xi}\left(1+a \xi^{2}\right)} \int_{0}^{t} \exp \left[-\frac{b \xi^{2}}{1+a \xi^{2}}(t-\tau)\right] \\
\times \sin \left[\sigma_{\xi}(t-\tau)\right] \widehat{f}(\xi, t) d \tau,
\end{gathered}
$$


where

$$
\sigma_{\xi}=\frac{\sqrt{a c \xi^{6}+\left(a+c-b^{2}\right) \xi^{4}+(1+a d) \xi^{2}+d}}{1+a \xi^{2}} .
$$

Hence, we have

$$
\begin{aligned}
|\widehat{w}(\xi, t)| & \leqslant C \int_{0}^{t}\left|\exp \left[\frac{-b \xi^{2}}{1+a \xi^{2}}(t-\tau)\right] \widehat{f}(\xi, t)\right| d \tau \\
& \leqslant C\left[\int_{0}^{t}|\widehat{f}(\xi, t)|^{2} d \tau\right]^{\frac{1}{2}}
\end{aligned}
$$

Using inequality (29) and the Parseval inequality leads to

$$
\begin{aligned}
& \int_{-\infty}^{+\infty}|\widehat{w}(\xi, t)|^{2} d \xi \leqslant \int_{-\infty}^{+\infty} \int_{0}^{t}|\widehat{f}(\xi, t)|^{2} d \tau d \xi \\
& \leqslant C \int_{0}^{t}\|\widehat{f}(\xi, t)\|_{L^{2}}^{2} d \tau \\
& \leqslant C \int_{0}^{t}\left\|w(x, t)\left(u^{(1)}(x, t)+u^{(2)}(x, t)\right)\right\|_{L^{2}}^{2} d \tau \\
& \leqslant C \int_{0}^{t} c_{\tau}\|w(x, t)\|_{L^{2}}^{2} d \tau .
\end{aligned}
$$

By Growall's inequality, we get $w(x, t)=0$ (in $\left.L^{2}\right)$. Using the continuity of functions $u^{1}(x, t)$ and $u^{2}(x, t)$ results in $u^{1}(x, t)=u^{2}(x, t)$. It completes the proof of uniqueness.

\subsection{Long time asymptotics}

In order to find the long time behavior of the constructed solution, we firstly determine a subtle asymptotic $\widehat{u}_{1}(t)$. Since $\widehat{u}_{1}(t)=\sum_{N=0}^{\infty} \varepsilon^{N+1} \widehat{\xi}_{1}^{(N)}(t)$, from (18) and (19), we get

$$
\begin{array}{r}
\widehat{\xi}_{1}^{(0)}(t)=e^{-\frac{b}{1+a} t}\left[A^{0} \cos (\sigma t)+B^{0} \sin (\sigma t)\right], \\
\widehat{\xi}_{1}^{(N)}(t)=e^{-\frac{b}{1+a} t}\left[A^{N} \cos (\sigma t)\right]+\left[B^{N} \sin (\sigma t)\right],
\end{array}
$$

where

$$
\begin{aligned}
A^{(0)} & =\varepsilon \widehat{\varphi}_{1} \\
B^{(0)} & =\frac{\varepsilon}{\sigma}\left(\frac{b}{1+a} \widehat{\varphi}_{1}+\widehat{\psi}_{1}\right), \\
\sigma & =\frac{\sqrt{a c+a+c-b^{2}+1+a d+d}}{1+a}, \\
A^{(N)} & =\frac{\beta-h}{\sigma(1+a)} \int_{0}^{t} e^{\frac{b}{1+a} \tau} \sin (\sigma \tau) S_{u_{n}}(\tau) d \tau, \\
B^{(N)} & =\frac{\beta-h}{\sigma(1+a)} \int_{0}^{t} e^{\frac{b}{1+a} \tau} \cos (\sigma \tau) S_{u_{n}}(\tau) d \tau, \\
S_{u_{n}}(t) & =\sum_{g=1}^{\infty} \sum_{j=1}^{N} \widehat{\xi}_{1+g}^{j-1}(t) \widehat{\xi}_{g}^{(N-j)}(t) N \geqslant 1,
\end{aligned}
$$

where $\widehat{\xi}^{(j)}(t), j=0,1, \ldots N-1$ are defined by $(18)$ and (19).

For $n \geqslant 2, N \geqslant 1$, using a method similar to that used in [8], it follows from (22) that there exists a positive number $\eta\left(0<\eta<\frac{3}{1+4 a}\right)$ such that

$$
\left|R_{A}^{(N)}(t)\right| \leqslant C e^{\frac{-\eta b t}{1+a}}, \quad\left|R_{B}^{(N)}(t)\right| \leqslant C e^{\frac{-\eta b t}{1+a}} .
$$

Hence, as $t$ is sufficiently large, we have proved that

$$
2 i \widehat{u}_{1}(t)=e^{\frac{-b t}{1+a}}[A \cos (\sigma t)+B \sin (\sigma t)],
$$

where

$$
\begin{aligned}
& A=2 i \sum_{N=0}^{\infty} \varepsilon^{N+1} A^{(N)}, \\
& B=2 i \sum_{N=0}^{\infty} \varepsilon^{N+1} B^{(N)},
\end{aligned}
$$

in which $A^{(N)}$ and $B^{(N)}$ are defined by (30), and the series $\mathrm{A}$ and $\mathrm{B}$ converge absolutely and uniformly for $\varepsilon \in\left[0, \varepsilon_{0}\right]$. Now, we have

$$
u(x, t)=2 i \widehat{u}_{1}(t) \sin x+R_{u}(x, t),
$$

where

$$
R_{u}(x, t)=2 i \sum_{n=2}^{\infty} \sin n x \sum_{N=1}^{\infty} \varepsilon^{N+1} \widehat{\xi}_{n}^{(N)}(t) .
$$

Using inequality (22) results in

$$
\begin{aligned}
& \left|R_{u}(x, t)\right| \leqslant \exp \left[\frac{-(1+\eta) b t}{1+a}\right] \\
& \times \sum_{N=1}^{\infty} C^{N} \varepsilon^{N+1}(N+1)^{-2} \sum_{n=2}^{\infty} n^{-6}, \\
& \leqslant C \exp \left[\frac{-(1+\eta) b t}{1+a}\right] .
\end{aligned}
$$

It derives from (32), (33) and (34) that formula (6) is valid. Thus, the proof of the theorem is complete.

\section{Conclusions}

In conclusion, we would like to return the issue of the smallness of initial data associated in problem (4). Although this assumption is needed to show the global-in-time existence for the problem, some equations admit global-in-time solutions for large initial data. Such solutions may have time decay due to dissipation or dispersion. It is the time decay that solutions will become small beginning from some $t=T$ and the asymptotics will be valid for the large initial data as $t$ tends to infinite. 


\section{Acknowledgment}

This work is supported by the Scientific Research Fund of Southwestern University of Finance and Economics (Grant No. 07YB53). The corresponding author's email address: laishaoy@swufe.edu.cn

\section{References}

[1] J. Boussinesq, Theorie des ondes et de remous qui se propagent le long d un canal recangulaier, et communiquant au liquide contene dans ce cannal des vitesses sensiblement pareilles de la surface au fond, J.Math.Pures Appl., 17: 55108, 1872.

[2] J. Bona and R. Sachs, Global existence of smooth solutions and stability of solitary waves for a generalized Boussinesq equation, Common.Math.Phys., 118: 12-29, 1998.

[3] P. Clarkson, New exact solutions of the Boussinesq equation , Eur.J.Appl.Maths., 1: 279-300, 1990.

[4] V.M. Galkin, D.E.Pelinovksy, and Yu. A. Stepanyants, The stru cture of the rational solution to the Boussinesq equation, Physica D, 80: 246255, 1995.

[5] R. Hirota, Solutions of the cassical Boussinesq equation and the spherical Boussinesq equation: The Wronskian Technique, J.Phy.Soc.Japan., 55: 2137-2150, 1986.

[6] A. Nakamura, Exact solitary wave solutions of the spherical Boussinesq equation, J.Phys. Japan, 54: 4111-4114, 1985.

[7] V.V. Varlamov, On the Initial boundary Value Problem for the damped Boussinesq equation, Discrete and Continuous Dynamical Systems, 3: 431-444, 1998.

[8] V.V. Varlamov,Eigenfuntion expansion meth and the long -time asymptotics for the damped Boussinesq equation, Discrete and Continuous Dynamical Systems, 7:675-702, 2001.

[9] S.Y. Lai, The asymptotic theory of solutions for a perturbed telegraph wave equation and its application, Appl.Math.Mech, 43(7): 657-662, 1997.

[10] S.Y. Lai and Q.L. Fu, The asymptotics theory of initial value problem for semilinear perturbed wave equation, Appl.Math.Mech, 24(1):82-91, 2003.

[11] N. Polat, Blow up of solutions for the damped Boussinesq equation, Journal of Physical Sciences, 60(7): 473-476, 2005. 